\title{
Greater coordination and harmonisation of European occupational cohorts is needed
}

\author{
Michelle C Turner, ${ }^{1,2,3,4}$ Ingrid Sivesind Mehlum ${ }^{5}$
}

Paid employment is an essential component of adult life and a major determinant of health. However, underemployment, long-term unemployment, poor working conditions and a lack of job security all negatively affect health, may hinder economic growth and further increase inequalities in the population. Occupational exposures are related to a significant proportion of diseases including cancer, cardiorespiratory diseases and musculoskeletal and mental disorders, among others. ${ }^{1}$ The demographic shift, with an ageing and increasingly diverse workforce, makes the impact of work on healthy ageing and disease prognosis a key issue. Rapid changes in employment patterns and exposures along with occupational restructuring and the increasing use of new technologies further increase the importance of research in occupational health. ${ }^{2}$

Europe currently has some of the most valuable occupational, industrial and population cohorts for aetiological research worldwide. However, relatively limited sample sizes of individual studies and lack of data harmonisation have meant that evidence of potential occupational hazards is often inconsistent and inconclusive, leading to delayed regulatory action. Better integration and coordination of these cohorts would improve the optimal exploitation of these resources, essential to underpin evidence-based interventions and policy.

The benefits of large-scale co-ordination can be seen in several recent initiatives. MODERNET, a 2010-2014 European Cooperation in Science and Technology (COST) action, created a network to develop new techniques for surveillance

\footnotetext{
${ }^{1}$ Barcelona Institute for Global Health (ISGlobal), Barcelona, Spain

${ }^{2}$ Universitat Pompeu Fabra (UPF), Barcelona, Spain ${ }^{3}$ CIBER Epidemiología y Salud Pública (CIBERESP), Madrid, Spain

${ }^{4}$ McLaughlin Centre for Population Health Risk Assessment, University of Ottawa, Ottawa, Ontario, Canada

${ }^{5}$ National Institute of Occupational Health (STAMI), Oslo, Norway
}

Correspondence to Dr Michelle C Turner, Barcelona Institute for Global Health (ISGlobal), Barcelona 08003, Spain; michelle.turner@isglobal.org of trends in occupational diseases and tracing new and emerging risks. Changes in incidence of occupational asthma, contact dermatitis, noise-induced hearing loss, carpal tunnel syndrome and upper limb musculoskeletal disorders were compared for the first time across diverse surveillance systems in 10 European countries. ${ }^{3}$ StanDerm (2012-2016) sought to create standards for the prevention and clinical management of occupational skin diseases. ${ }^{4}$ The Environmental Health Risks in European Birth Cohorts (ENRIECO) ${ }^{5}$ and Developing a Child Cohort Research Strategy for Europe (CHICOS) (www. chicosproject.eu) projects, and related birth cohort inventory (www.birthcohorts. net), also led to substantial advancements in child health research.

The Network on the Coordination and Harmonisation of European Occupational Cohorts (OMEGA-NET) (http://www. cost.eu/COST_Actions/ca/CA16216) was recently created, currently with participants from 28 European countries and one international partner. OMEGA-NET is a 4-year action (2017-2021) supported by the EU-funded COST Association. It seeks to optimise the use of occupational, industrial and population cohorts at the European level by advancing (1) collaboration of cohorts with extensive contemporary information on employment and occupational exposures, (2) co-ordination and harmonisation of both new and existing occupational exposure assessment efforts, and (3) facilitation of an integrated research strategy for occupational health in Europe, with the aim to extend globally.

More specifically, we seek to inventory numerous cohorts with occupational information in Europe (an initial evaluation indicates at least 63 major individual prospective and retrospective cohorts as well as several large record linkage studies with occupational information on over 30 million workers), implement an online interactive tool with detailed information on existing cohorts, facilitate work on harmonisation of existing occupational exposure and health outcome information and create and pilot-test new protocols for data collection, and connect scientific communities and relevant stakeholders.
OMEGA-NET will focus initially on several research areas including, but not limited to (1) healthy ageing and work participation including that of chronically ill workers, (2) working hours with emphasis on shift work and alternate work hours, (3) employment patterns in the young with an emphasis on young employed women, (4) occupational skin diseases, (5) work-related psychosocial determinants of mental health and (6) precarious work.

OMEGA-NET will enhance the scientific output from individual studies and facilitate pooled studies, data sharing, and transfer of tools and skills to make greater and more efficient use of existing cohorts. It will also provide a unique resource for hypothesis-driven research, and further support recent calls for the increased use of prospective cohorts in aetiological research on workplace exposures. ${ }^{6}$ Through facilitating standardisation of both new and existing data, OMEGA-NET will provide an opportunity to investigate the causes of variability in research findings between studies and eventually address those related to methodological inconsistencies. By combining data from multiple cohorts, it may be possible to address research questions with greater statistical power, particularly when looking at interactions between multiple risk factors, subgroup effects and rare exposures/outcomes, and exploit between-countries differences (see for example the International Nuclear Workers Study, ${ }^{7}$ the AGRICOH Consortium of Agricultural Cohorts ${ }^{8}$ and the Individual-Participant Data Meta-Analysis in Working Populations consortium ${ }^{9}$ ). It can also contribute to evaluating evidence and classifying carcinogens. ${ }^{10}$

Exposure assessment will be facilitated through standardised and transparent approaches in both new and existing studies. Established large European population-based cohorts have so far been under-used for occupational epidemiology, mainly due to the lack of exposure information, including comprehensive job-exposure matrices (JEMs). Occupational exposure assessment tools, such as existing databases and JEMs, will be inventoried and brought together to build an open exposure assessment resource. Where applicable, JEMs will be compared and validated against available exposure data.

It is important to note, however, that coordination and harmonisation is not without challenges, often requiring lengthy processes of comparison, validation, data reduction and compromise, the demands of which should not be 
understated. $^{11} 12$ Differences between studies may also be greater than originally anticipated and careful interpretation of results required. ${ }^{7}{ }^{12}$ Although we will focus on selected high priority exposure/ outcome variables of interest, time and resources will likely impact the work. Other potential limitations may include the inventory of occupational cohorts that are either small, poorly documented, based in private industry or historical; however, we seek primarily to improve coordination among active, or potentially active, cohorts for future use. A balance will also be sought in terms of the amount of data captured in the inventory to maximise its usefulness while minimising the burden of data collected from each cohort. Although new protocols for data collection will be prepared and disseminated, their use will depend on implementation in individual cohorts.

Overall, OMEGA-NET will provide a foundation for an enhanced evidence base for the identification of health risks and gains related to occupation and employment and strengthened occupational health policies in Europe. Substantial advancements in occupational health research will be realised with a coordinated European, as well as international, approach to research and dissemination.

Contributors Both authors contributed to the conception, writing, revision and finalisation of the editorial.

Funding This publication is based upon work from COST Action CA16216, supported by COST (European Cooperation in Science and Technology). MCT is supported by the Departament de Salut, Generalitat

de Catalunya (SLT002/16/00232) . ISGlobal is a member of the CERCA Programme, Generalitat de Catalunya.

Competing interests None declared.

Patient consent Not required.

Provenance and peer review Commissioned; internally peer reviewed.

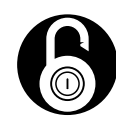

\section{OPEN ACCESS}

Open access This is an Open Access article distributed in accordance with the Creative Commons Attribution Non Commercial (CC BY-NC 4.0) license, which permits others to distribute, remix, adapt, build upon this work non-commercially, and license their derivative works on different terms, provided the original work is properly cited and the use is non-commercial. See: http:// creativecommons.org/licenses/by-nc/4.0/

(C) Article author(s) (or their employer(s) unless otherwise stated in the text of the article) 2018. All rights reserved. No commercial use is permitted unless otherwise expressly granted.

\section{(D) Check for updates}

To cite Turner MC, Mehlum IS. Occup Environ Med 2018;75:475-476.

Received 10 January 2018

Revised 17 March 2018

Accepted 23 April 2018

Published Online First 7 May 2018

Occup Environ Med 2018;75:475-476.

doi:10.1136/oemed-2017-104955

\section{REFERENCES}

1 GBD 2016 Risk Factors Collaborators. Global, regional, and national comparative risk assessment of 84 behavioural, environmental and occupational, and metabolic risks or clusters of risks, 1990-2016: a systematic analysis for the Global Burden of Disease Study 2016. Lancet 2017;390:1345-422.

2 Eurofound. Sixth European Working ConditionsOverview report (2017 update). Luxembourg: Publications Office of the European Union, 2017.

3 Stocks SJ, McNamee R, van der Molen HF, et al. Trends in incidence of occupational asthma, contact dermatitis, noise-induced hearing loss, carpal tunnel syndrome and upper limb musculoskeletal disorders in European countries from 2000 to 2012. Occup Environ Med 2015;72:294-303.

4 Alfonso JH, Bauer A, Bensefa-Colas L, et al. Minimum standards on prevention, diagnosis and treatment of occupational and work-related skin diseases in Europe-position paper of the COST Action StanDerm (TD 1206). J Eur Acad Dermatol Venereol 2017;31(Suppl. 4):31-43.

5 Vrijheid M, Casas M, Bergström A, et al. European birth cohorts for environmental health research. Environ Health Perspect 2012;120:29-37.

6 Blair A, Hines CJ, Thomas KW, et al. Investing in prospective cohorts for etiologic study of occupational exposures. Am J Ind Med 2015;58:113-22.

7 Hamra GB, Richardson DB, Cardis E, et al. Cohort profile: the International Nuclear Workers Study (INWORKS). Int J Epidemiol 2016;45:693-9.

8 Brouwer M, Schinasi L, Beane Freeman LE, et al. Assessment of occupational exposure to pesticides in a pooled analysis of agricultural cohorts within the AGRICOH consortium. Occup Environ Med 2016;73:359-67.

9 Dragano N, Siegrist J, Nyberg ST, et al. Effort-reward imbalance at work and incident coronary heart disease: a multicohort study of 90,164 Individuals. Epidemiology 2017;28:619-26.

10 DDT. International Agency for Research on Cancer. 113. Lyon, France: Lindane, and 2,4-D, 2015.

11 Glass DC, Armstrong TW, Pearlman ED, et al. Ensuring comparability of benzene exposure estimates across three nested case-control studies in the petroleum industry in support of a pooled epidemiological analysis. Chem Biol Interact 2010;184:101-11.

12 Bao SS, Kapellusch JM, Garg A, et al. Developing a pooled job physical exposure data set from multiple independent studies: an example of a consortium study of carpal tunnel syndrome. Occup Environ Med 2015;72:130-7. 